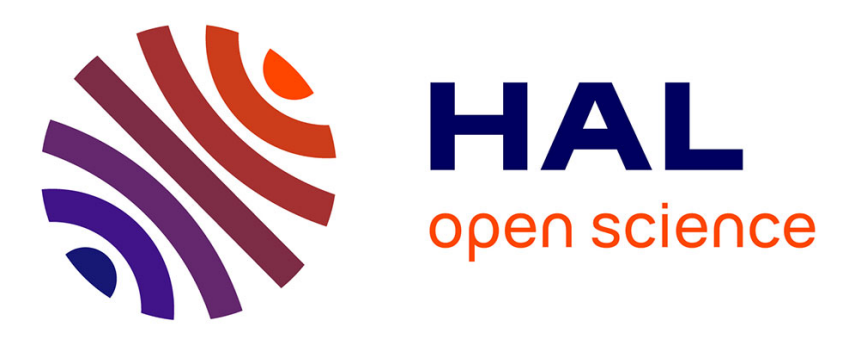

\title{
On linear MMSE based turbo-equalization of nonlinear Volterra channels
}

\author{
Bouchra Benammar, Nathalie Thomas, Charly Poulliat, Marie-Laure \\ Boucheret, Mathieu Dervin
}

\section{- To cite this version:}

Bouchra Benammar, Nathalie Thomas, Charly Poulliat, Marie-Laure Boucheret, Mathieu Dervin. On linear MMSE based turbo-equalization of nonlinear Volterra channels. IEEE International Conference on Acoustics, Speech, and Signal Processing - ICASSP 2013, May 2013, Vancouver, Canada. pp. 47034707. hal-01150348

\author{
HAL Id: hal-01150348 \\ https://hal.science/hal-01150348
}

Submitted on 11 May 2015

HAL is a multi-disciplinary open access archive for the deposit and dissemination of scientific research documents, whether they are published or not. The documents may come from teaching and research institutions in France or abroad, or from public or private research centers.
L'archive ouverte pluridisciplinaire HAL, est destinée au dépôt et à la diffusion de documents scientifiques de niveau recherche, publiés ou non, émanant des établissements d'enseignement et de recherche français ou étrangers, des laboratoires publics ou privés. 


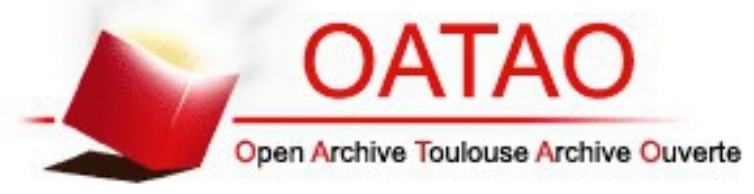

\section{Open Archive TOULOUSE Archive Ouverte (OATAO)}

OATAO is an open access repository that collects the work of Toulouse researchers and makes it freely available over the web where possible.

This is an author-deposited version published in : http://oatao.univ-toulouse.fr/ Eprints ID : 12480

To link to this article : DOI :10.1109/ICASSP.2013.6638552

URL : http://dx.doi.org/10.1109/ICASSP.2013.6638552

To cite this version : Benammar, Bouchra and Thomas, Nathalie and Poulliat, Charly and Boucheret, Marie-Laure and Dervin, Mathieu $\underline{O n}$ linear MMSE based turbo-equalization of nonlinear Volterra channels. (2013) In: IEEE International Conference on Acoustics, Speech, and Signal Processing - ICASSP 2013, 26 May 2013 - 31 May 2013 (Vancouver, Canada).

Any correspondance concerning this service should be sent to the repository administrator: staff-oatao@,listes-diff.inp-toulouse.fr 


\title{
ON LINEAR MMSE BASED TURBO-EQUALIZATION OF NONLINEAR VOLTERRA CHANNELS
}

\author{
Bouchra Benammar*, Nathalie Thomas*, Charly Poulliat*, Marie-Laure Boucheret* and Mathieu Dervin ${ }^{\dagger}$ \\ * University of Toulouse, INPT-ENSEEIHT/IRIT \\ $\dagger$ Thales Alenia Space, Toulouse \\ Email: \{bouchra.benammar, nathalie.thomas, charly.poulliat, marie-laure.boucheret\}@enseeiht.fr, \\ mathieu.dervin@thalesaleniaspace.com
}

\begin{abstract}
This article deals with Minimum Mean Square Error (MMSE) turbo equalization of nonlinear interference using a volterra series decomposition of the underlying nonlinear channel. Although it has been often argued that linear MMSE based equalization is unsuited for cancelling nonlinear interference, we show that this common belief is not true in a strict sense. By a proper derivation of the linear based MMSE soft equalizer, we are able to show that the underlying structure of the equalizer is equivalent to a Soft Interference Canceller (SIC) treating both the linear and nonlinear interference. Based on these results, approximations are provided for lowering the computational complexity. Links to previously proposed "nonlinear" SIC are emphasized showing that the previously proposed structures are nothing but approximations of a linear MMSE receiver applied to nonlinear ISI channels. Simulations show that significant improvements can be achieved by using the proposed exact and approximate MMSE based turbo-equalizers.
\end{abstract}

Index Terms - iterative equalization, volterra series, nonlinear interference

\section{INTRODUCTION}

Satellite transmissions are impacted by nonlinear distortions due to the on-board High Power Amplifier (HPA) stage. For the sake of a better energy efficiency, these HPAs -Travelling Wave Tubes (TWT) or Solid State amplifiers (SS) -are driven as close as possible from their saturation. A "back-off" is however usually required so as to guarantee the best trade-off between the useful signal power and the nonlinear distortion effects. These effects are all the more significant when high modulation orders are used. Thus, the use of high order modulations such as those proposed in DVB-S2 standard [1] for instance, calls for nonlinearities compensation in the transmission chain.

Nonlinear satellite distortions can be treated either at the transmitter with the so-called pre-compensation/pre-distortion techniques or at the receiver using equalization. In this paper, we are mainly interested in iterative equalization techniques. Several channel models have been investigated to cope with nonlinear interference.

A first approach was introduced in [2] [3] where the nonlinear channel was considered as an additive gaussian noise associated with a signal attenuation called warping. This approximation is no longer accurate for high order mutli-level modulations since outer rings constellation symbols experience stronger distortions that inner rings symbols. Yet, the most common approach to model the nonlinear distortions is the use of the Volterra series representations originally proposed by [4]. The investigated nonlinear satellite channel model is based on a Volterra series representation. When perfect knowledge of the channel is available at the receiver, Maximum A Postertiori (MAP)-based equalization techniques or Maximum Likelihood Sequence Detection (MLSD) show optimal performance based on the trellis representation of the equivalent nonlinear channel as investigated in $[5,6]$. However, their induced complexity (exponential in the channel memory) renders their use at the receiver rather difficult for high order modulations and long memory channels.

Therefore a great deal of research has been dedicated to the derivation of low complexity receivers. Inspired by the turbo principle [7][8][9], several attempts have been made to derive low complexity equalization and decoding schemes referred to as turbo-equalizers. They have shown to exhibit improved performance compared to noniterative receivers with a fair trade-off between performance and complexity. In [10], an iterative receiver is presented consisting of a factor graph detector with a complexity linear in the channel memory. Yet, most of existing turbo receivers for the nonlinear channel are based on MMSE equalization imposing a predetermined nonlinear soft interference cancelling structure mainly inspired by the structure proposed by [9].

In this article, we are interested in having insights for the linear MMSE-based turbo-equalization of nonlinear channels. Although cyclically studied, MMSE based iterative equalization has not been studied so far in a systematic manner as it has been the case for the linear ISI channel [7][11]. It has been argued in [12] [13] that linear MMSE equalization would not allow cancelling the nonlinear interference. However, such statement seems to be based on the formulas of the linear MMSE equalizer derived for a linear channel. The main contribution of this article is to show that the linear MMSE estimator derived properly for the Volterra channel induces a non linear SIC able to cancel both linear and nonlinear interference. Besides, it can be shown that the "nonlinear" ISI canceller proposed in [12] is actually a special case of the linear MMSE applied to the Volterra channel. Moreover, in [13], authors derive a "nonlinear" low complexity SIC based on a particular channel decomposition which does not cancel all the nonlinear interference. As a result, the proposed turbo-equalizer shows poor performance when compared to a SIC treating only linear interference for the almost all investigated scenarios.

The remainder of this paper is organised as follows: first, we present the Volterra channel model. Then, we derive general formulas for the Volterra-MMSE turbo-equalizer as well as some time-invariant approximations. Finally, we investigate the performances of the different MMSE implementations before ending with some conclusions. 


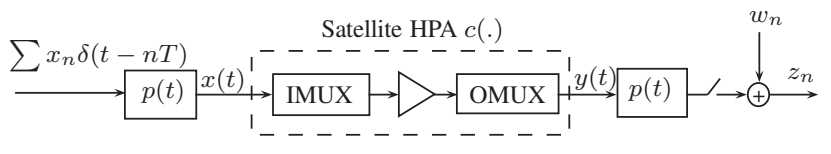

Fig. 1. Block diagram of a satellite communication channel

\section{NONLINEAR VOLTERRA CHANNEL MODEL}

The satellite transponder's HPA is usually represented by an IMUX (Input MUltipleXing filter) followed by a memoryless nonlinear amplifier and an OMUX (Output MUltipleXing filter). The constituent IMUX filter aims at removing adjacent channel interferences, whereas the OMUX filters the out-of band frequencies ensuing from the spectral enlargement due to the nonlinear processing within the amplifier. According to [4], we can assume that the satellite transponder corresponds to a bandpass HPA whose transfer function $c$ only accounts for the module of the signal $x$ to be amplified:

$$
\begin{aligned}
y & =c(|x|) \exp (j \varphi(x)) \\
& =A(|x|) \exp (j \Phi(|x|)+\varphi(x))
\end{aligned}
$$

where $y$ is the amplified signal and $A($.$) and \Phi($.$) are called AM/AM$ and $\mathrm{AM} / \mathrm{PM}$ characteristics.

Much of satellite HPA models found in literature rely on Saleh's model [14] who proposed an analytical frequency independent model for power amplifiers. Another model was presented by [4] et al, who proved that due to the bandpass nature of the satellite transponder, its transfer function could be decomposed into series containing only odd terms:

$$
c(|x|)=\sum_{k} \gamma_{2 k+1}|x|^{2 k+1}
$$

This decomposition is the baseline for a Volterra description of the nonlinear channel as will be shown later in this section.

Let us consider the satellite communication model depicted in fig. 1. Let $x(t)$ be the baseband equivalent of the transmitted signal:

$$
x(t)=\sum_{n} x_{n} p(t-n T),
$$

where $x_{n}$ denote independent identically distributed symbols drawn from an M-ary alphabet, $p(t)$ represents the normalized shaping filter and $T$ is the symbol duration. The amplified signal $y(t)$ can be written as:

$$
y(t)=c(|x(t)|) \exp (j \varphi(x(t))),
$$

and is followed at the receiver by a downlink noise which is supposed to be zero mean additive circular white Gaussian process with variance $\sigma_{w}^{2}$. Matched filtering and sampling at $t=t_{0}+n T$ leads to the so-called Volterra channel given by:

$$
\begin{aligned}
z_{n} \triangleq z\left(t_{0}+n T\right)= & \sum_{m=0}^{v_{m}} \sum_{n_{1}} \ldots \sum_{n_{2 m+1}} x_{n-n_{1}} \ldots x_{n-n_{m+1}} \\
& x_{n-n_{m+2}}^{*} \ldots x_{n-n_{2 m+1}}^{*} h_{n_{1}, \ldots, n_{2 m+1}}+w_{n}
\end{aligned}
$$

where $v_{m}$ determines the decomposition order of the Volterra series, $h_{n_{1}, \ldots, n_{2 m+1}}$ are called Volterra kernels and $w_{n}$ is the filtered additive noise at time $t_{0}+n T$.

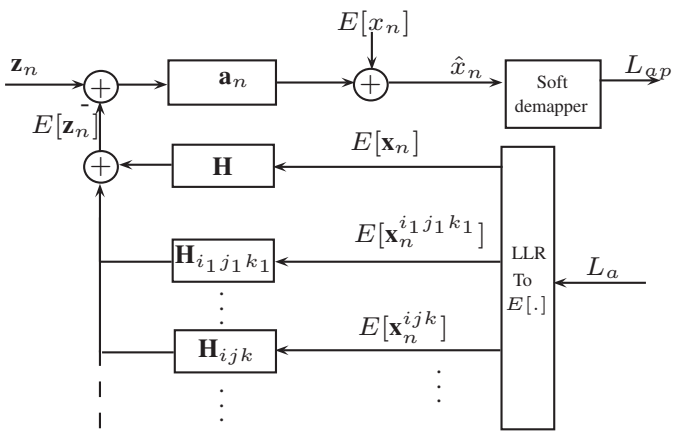

Fig. 2. Block diagram of the MMSE equalizer

For the remaining of this article and for ease of presentation, the above discrete time Volterra channel model will be truncated up to the 3rd order:

$$
z_{n}=\sum_{i=-L_{02}}^{L_{01}} h_{i} x_{n-i}+\sum_{i} \sum_{j} \sum_{k} h_{i j k} x_{n-i} x_{n-j} x_{n-k}^{*}+w_{n}
$$

where $L_{02}$ and $L_{01}$ define the channel linear ISI length.

\section{LINEAR VOLTERRA-MMSE TURBO EQUALIZATION}

In this section, we derive the exact formulas for MMSE based soft equalization. Let us define the following notations:

$$
\begin{aligned}
& \mathbf{z}_{n} \triangleq\left[z_{n-N_{1}}, \ldots, z_{n+N_{2}}\right]^{T} \\
& \mathbf{x}_{n} \triangleq\left[x_{n-N_{1}-L_{01}}, \ldots, x_{n+N_{2}+L_{02}}\right]^{T} \\
& \mathbf{w}_{n} \triangleq\left[w_{n-N_{1}}, \ldots, w_{n+N_{2}}\right]^{T}
\end{aligned}
$$

It follows that:

$$
\mathbf{z}_{n}=\mathbf{H} \mathbf{x}_{n}+\sum_{i} \sum_{j} \sum_{k} \mathbf{H}_{i j k} \mathbf{x}_{n}^{i j k}+\mathbf{w}_{n}
$$

where $\mathbf{H} \triangleq$ Toeplitz $\left(\left[h_{L_{01}} \ldots h_{0} \ldots h_{-L_{02}}, \mathbf{0}_{1 \times N_{1}+N_{2}}\right]\right)$ is the linear convolution matrix having $\left[h_{L_{01}} \ldots h_{0} \ldots h_{-L_{02}}, \mathbf{0}_{1 \times N_{1}+N_{2}}\right]$ at its first row; $\mathbf{0}_{1 \times N_{1}+N_{2}}$ is the $\left(1 \times N_{1}+N_{2}\right)$ all zero vector; $\mathbf{H}_{i j k}=h_{i, j, k} \mathbb{I}_{N}$ are the nonlinear ISI matrices where $N=$ $N_{1}+N_{2}+1$ and $\mathbb{I}_{N}$ is the $N \times N$ identity matrix; and $\mathbf{x}_{n}^{i j k}$ are the third order ISI terms:

$$
\mathbf{x}_{n}^{i j k} \triangleq\left[\begin{array}{c}
x_{n-N_{1}-i} x_{n-N_{1}-j} x_{n-N_{1}-k}^{*} \\
\vdots \\
x_{n+N_{2}-i} x_{n+N_{2}-j} x_{n+N_{2}-k}^{*}
\end{array}\right]
$$

\section{3.a. Exact MMSE implementation}

The linear MMSE equalizer consists of an affine estimation of the received signal [11] [15]:

$$
\hat{x}_{n}=\mathbf{a}_{n}^{H} \mathbf{z}_{n}+b_{n}
$$

which computes estimates that minimize the mean square error with the transmitted symbols $E\left[\left|\hat{x}_{n}-x_{n}\right|^{2}\right]$.The time variant MMSE coefficients are given as follows:

$$
\left\{\begin{array}{l}
\mathbf{a}_{n}=\operatorname{Cov}\left(\mathbf{z}_{n}, \mathbf{z}_{n}\right)^{-1} \operatorname{Cov}\left(\mathbf{z}_{n}, x_{n}\right) \\
b_{n}=E\left[x_{n}\right]-\mathbf{a}_{n}^{H} E\left[\mathbf{z}_{n}\right]
\end{array}\right.
$$


where $\operatorname{Cov}(\mathbf{x}, \mathbf{y}) \triangleq E\left[\mathbf{x y}^{H}\right]-E[\mathbf{x}] E\left[\mathbf{y}^{H}\right]$, leading to:

$$
\hat{x}_{n}=\mathbf{a}_{n}^{H}\left(\mathbf{z}_{n}-E\left[\mathbf{z}_{n}\right]\right)+E\left[x_{n}\right]
$$

where

$$
E\left[\mathbf{z}_{n}\right]=\mathbf{H} E\left[\mathbf{x}_{n}\right]+\sum_{i} \sum_{j} \sum_{k} \mathbf{H}_{i j k} E\left[\mathbf{x}_{n}^{i j k}\right]
$$

We recognise in equation (9) and fig.2 the structure of a soft interference canceller where linear and nonlinear ISI terms appearing in $E\left[\mathbf{z}_{n}\right]$ are cancelled. Note that in order not to cancel the current symbol to be estimated, the apriori LLR $\left(L_{a}\right)$ for the $n^{\text {th }}$ symbol should not be taken into account in the linear ISI terms by considering $E\left[x_{n}\right]=0$ and $\operatorname{var}\left(x_{n}\right) \triangleq v_{n}=1$ only for the linear ISI terms. Equations (8) of the MMSE equalizer can then be detailed as follows:

$$
\begin{aligned}
\operatorname{Cov}\left(\mathbf{z}_{n}, \mathbf{z}_{n}\right) & =\sigma_{w}^{2} \mathbb{I}_{N}+\mathbf{H} \operatorname{Cov}\left(\mathbf{x}_{n}, \mathbf{x}_{n}\right) \mathbf{H}^{H}+\left(1-v_{n}\right) \mathbf{h}_{n} \mathbf{h}_{n}^{H} \\
& +\sum_{i, j, k} \mathbf{H}_{i j k} \operatorname{Cov}\left(\mathbf{x}_{n}^{i j k}, \mathbf{x}_{n}\right) \mathbf{H}^{H} \\
& +\sum_{i, j, k} \mathbf{H} \operatorname{Cov}\left(\mathbf{x}_{n}, \mathbf{x}_{n}^{i j k}\right) \mathbf{H}_{i j k}^{H} \\
& +\sum_{i, j, k} \sum_{i^{\prime}, j^{\prime}, k^{\prime}} \mathbf{H}_{i j k} \operatorname{Cov}\left(\mathbf{x}_{n}^{i j k}, \mathbf{x}_{n}^{i^{\prime} j^{\prime} k^{\prime}}\right) \mathbf{H}_{i^{\prime} j^{\prime} k^{\prime}}^{H} \\
\operatorname{Cov}\left(\mathbf{z}_{n}, x_{n}\right)= & \mathbf{H C o v}\left(\mathbf{x}_{n}, x_{n}\right)+\left(1-v_{n}\right) \mathbf{h}_{n} \\
& +\sum_{i, j, k} \mathbf{H}_{i j k} \operatorname{Cov}\left(\mathbf{x}_{n}^{i j k}, x_{n}\right) \\
E\left[\mathbf{z}_{n}\right]= & \mathbf{H} E\left[\mathbf{x}_{n}\right]-\mathbf{h}_{n} E\left[x_{n}\right]+\sum_{i, j, k} \mathbf{H}_{i j k} E\left[\mathbf{x}_{n}^{i j k}\right]
\end{aligned}
$$

where $\quad \mathbf{h}_{n}=\mathbf{H} \times\left[\mathbf{0}_{1 \times N_{1}+L_{01}}, 1, \mathbf{0}_{1 \times N_{2}+L_{02}}\right]^{T}$.

To obtain the exact MMSE coefficients one needs to compute expectations of products of 3,4 and 6 symbols and symbols conjugates at different time instants as mentioned in [4]. Due to the presence of the interleaver between the decoder and the equalizer, symbols can be considered to be mutually independent. Thus, we can write the average of a product of $p$ symbols and $q-p$ symbol conjugates as follows:

$$
\begin{aligned}
& E\left[x_{n-i_{1}} x_{n-i_{2}} \ldots x_{n-i_{p}} x_{n-i_{p+1}}^{*} \ldots x_{n-i_{q}}^{*}\right] \\
& =\prod_{j} E\left[x_{n-i_{j}}^{v_{j}} x_{n-i_{j}}^{* v_{j}^{*}}\right] \\
& =\prod_{j} \sum_{m=1}^{M} s_{m}^{v_{j}} s_{m}^{* v_{j}^{*}} P\left[x_{n-i_{j}}=s_{m}\right]
\end{aligned}
$$

where $v_{j}\left(v_{j}^{*}\right)$ represents the number of occurrences of symbol $x_{n-i_{j}}\left(x_{n-i_{j}}^{*}\right)$ in the product average, and $s_{m} m \in[1 \ldots M]$ is the $m^{t h}$ constellation symbol which probability is computed as:

$$
P\left(x_{n}=s_{m}\right)=\prod_{i=1}^{\log _{2}(M)} P\left(c_{n, i}=s_{m, i}\right)
$$

where $c_{n, i}$ is the $i^{t h}$ coded bit of the time $n$ symbol $x_{n}$ and $s_{m, i}$ is the $i^{\text {th }}$ bit of constellation symbol $s_{m}$.
The coded bits probabilities can be computed from the input Log-Likelihood Ratios (LLRs) $L_{a}$ from the decoder as:

$$
L_{a}\left(c_{n, i}\right)=\ln \frac{P\left(c_{n, i}=0\right)}{P\left(c_{n, i}=1\right)}
$$

Computing the exact terms of (8) at each equalization step, in addition to matrix inversions imply a significant computational complexity. However, since there exist common sub-matrices between successive time instants covariance matrices in (11) we can resort to fast recursive computations as described in [11].

\section{3.b. Time-invariant approximate MMSE coefficients}

An alternative to the computational complexity of the exact MMSE equalizer would be the use of time-invariant approximations of the MMSE coefficients. Two approximations can be proposed:

\section{3.b.1. No-Apriori (NA) MMSE approximate implementation}

In this first approximation, the MMSE coefficients are computed assuming no a priori knowledge is available at the equalizer, which is the case for the first turbo-iteration. In this case, $E\left[x_{n}\right]=0$ and $v_{n}=1 \forall n$. However, due to the nonlinear ISI terms, no simplified expression of the MMSE coefficients can be computed, since it depends on the modulation order, the channel coefficients and the type of modulation (PSK, QAM, APSK,...). When the symbols are drawn from a constant modulus modulation, the channel 3rd order kernels indexes $(i, j, k)$ verify that $i \neq k$ and $j \neq k$, since otherwise they would contribute to the linear ISI part. Thus, it can be shown for a modulation of order $M$ higher than 2 that:

$$
\begin{array}{ll}
\forall n & \forall(i, j, k) \quad E\left[\mathbf{x}_{n}^{i j k}\right]=\mathbf{0}_{N \times 1} \\
\forall n & \forall(i, j, k) \operatorname{Cov}\left(\mathbf{x}_{n}, \mathbf{x}_{n}^{i j k}\right)=\mathbb{O}_{N+L_{01}+L_{02} \times N} \\
\forall n & \forall(i, j, k) \operatorname{Cov}\left(\mathbf{x}_{n}^{i j k}, \mathbf{x}_{n}\right)=\mathbb{O}_{N \times N+L_{01}+L_{02}} \\
\forall n & \forall(i, j, k) \neq\left(i^{\prime}, j^{\prime}, k^{\prime}\right) \operatorname{Cov}\left(\mathbf{x}_{n}^{i j k}, \mathbf{x}_{n}^{i^{\prime} j^{\prime} k^{\prime}}\right)=\mathbb{O}_{N} \\
\forall n & \forall(i, j, k) \operatorname{Cov}\left(\mathbf{x}_{n}^{i j k}, \mathbf{x}_{n}^{i j k}\right)=\mathbb{I}_{N}
\end{array}
$$

Leading to simplified NA-MMSE coefficients:

$$
\mathbf{a}_{N A}=\left(\sigma_{w}^{2} \mathbb{I}_{N}+\mathbf{H} \mathbf{H}^{H}+\sum_{(i, j, k) \in \mathcal{T}}\left|h_{i j k}\right|^{2} \mathbb{I}_{N}\right)^{-1} \mathbf{h}_{n}
$$

These NA-coefficients are strictly equivalent to the ones proposed in [12]. The previous result can be generalized to APSK modulations if we assume that APSK can be approximated by constant amplitude modulations.

\section{3.b.2. Low complexity approximate implementation}

In this second implementation, we investigate another time invariant MMSE implementation introduced in [7]. The idea is to compute the MMSE filter that minimises $\frac{1}{L} \sum_{n=0}^{L-1} E\left[\left|\hat{x}_{n}-x_{n}\right|^{2}\right]$. The optimal solution becomes :

$$
\mathbf{a}_{L C}=\left(\frac{1}{L} \sum_{n=0}^{L-1} \operatorname{Cov}\left(\mathbf{z}_{n}, \mathbf{z}_{n}\right)\right)^{-1}\left(\frac{1}{L} \sum_{n=0}^{L-1} \operatorname{Cov}\left(\mathbf{z}_{n}, x_{n}\right)\right)
$$


Table 1. Test channel Volterra kernels

\begin{tabular}{|l|l|}
\hline 1st order kernels & 3rd order kernels \\
\hline \hline$h_{0}=0.8529+0.4502 i$ & $h_{002}=0.1091-0.0615 i$ \\
$h_{1}=0.0881-0.0014 i$ & $h_{330}=0.0503-0.0503 i$ \\
$h_{2}=-0.0336-0.0196 i$ & $h_{001}=0.0979-0.0979 i$ \\
$h_{3}=0.0503+0.0433 i$ & $h_{003}=-0.1119-0.0252 i$ \\
& $h_{110}=-0.0280-0.0475 i$ \\
\hline
\end{tabular}

Thus $\frac{1}{L} \sum_{n=0}^{L-1} \operatorname{Cov}\left(\mathbf{x}_{n}, \mathbf{x}_{n}\right)$ becomes a diagonal matrix and can be further simplified by assuming that side effects are negligible, which results in $\frac{1}{L} \sum_{n=0}^{L-1} \operatorname{Cov}\left(\mathbf{x}_{n}, \mathbf{x}_{n}\right)=v \mathbb{I}_{N}$ where $v=\frac{1}{L} \sum_{n=0}^{L-1} \operatorname{var}\left(x_{n}\right)$.

As for the third order covariances matrices, it can only be shown that they have a Toeplitz structure. The computational complexity is slightly reduced since matrix inversion is done once for every $L$ equalized symbols.

\section{3.c. Soft demapper}

In order to map the output of the equalizer to code LLRs, we define the residual equalizer output error $e_{n}=\hat{x}_{n}-x_{n}$ [7]. Computing the distribution of the estimation error instead of the distribution of $\hat{x}_{n}$ given $x_{n}$ turns out to be a practical choice, since one needs not to track occurrences of $x_{n}$ in third order covariances terms in (11). For practical considerations, this error is assumed to be Gaussian. The residual error has mean $E\left[e_{n}\right]=0$ and variance $\operatorname{var}\left(e_{n}\right)=1+\operatorname{Cov}\left(\hat{x}_{n}, \hat{x}_{n}\right)-2 R_{e}\left(\operatorname{Cov}\left(\hat{x}_{n}, x_{n}\right)\right)$.

For the exact MMSE implementation, it can be shown that $\operatorname{var}\left(e_{n}\right)=$ $1-\operatorname{Cov}\left(x_{n}, \mathbf{z}_{n}\right) \mathbf{a}_{n}$.

As for the NA-MMSE implementation, computing the exact error variance is more computationally complex than for the exact MMSE. Hence, we shall refer to it as implementation-a. Authors in [12] presented a simplified NA-MMSE error-variance which neglects the contribution of third order Volterra terms. This simplification will be referred to as implementation-b. Although being less computationally complex than implementation-a, the proposed simplification would provide inaccurate LLRs at high SNR values, since nonlinear terms can no longer be neglected.

The equalizer output $\hat{x}_{n} \sim \mathcal{N}\left(x_{n}, \operatorname{var}\left(e_{n}\right)\right)$ and the output $a$ posteriori LLR $L_{a p}$ can be written as:

$$
\begin{aligned}
& L_{a p}\left(c_{n, i} \mid \hat{x}_{n}\right) \triangleq \ln \frac{P\left(c_{n, i}=0 \mid \hat{x}_{n}\right)}{P\left(c_{n, i}=1 \mid \hat{x}_{n}\right)} \\
& =\ln \frac{\sum_{s_{j}: s_{j i}=0}\left(\exp \left(\frac{-\left|\hat{x}_{n}-s_{j}\right|^{2}}{\operatorname{var}\left(e_{n}\right)}\right)\right) \prod_{k} P\left(c_{n, k}=s_{j, k}\right)}{\sum_{s_{j}: s_{i j}=1}\left(\exp \left(\frac{-\left|\hat{x}_{n}-s_{j}\right|^{2}}{\operatorname{var}\left(e_{n}\right)}\right)\right) \prod_{k} P\left(c_{n, k}=s_{j, k}\right)}
\end{aligned}
$$

The a posteriori LLRs are made extrinsic $\left(L_{e}\right)$ by subtracting the a priori probability fed by the decoder: $L_{e}\left(c_{n, i}\right)=L_{a p}\left(c_{n, i} \mid \hat{x}_{n}\right)-$ $L_{a}\left(c_{n, i}\right)$.

\section{SIMULATION RESULTS}

This section provides results of the performance of the above mentioned MMSE equalizers in a typical nonlinear satellite channel. In practice, only significant channel gains $h_{i j k}$ in (6) are considered in the Volterra decomposition of a satellite channel. The test channel we are considering is the one proposed by [12] which was derived

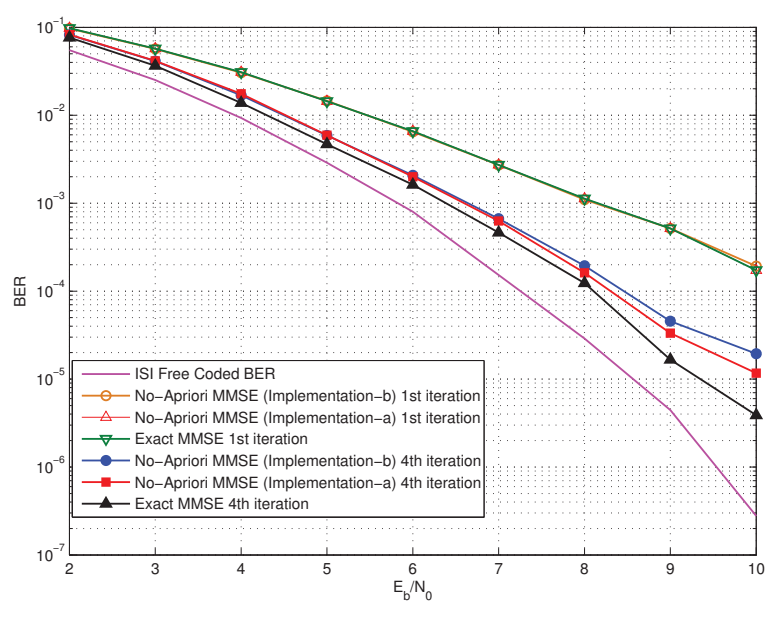

Fig. 3. BER performance of 8PSK MMSE-based linear equalizer implementations.

from [4] but with stronger nonlinear ISI (cf Table 1).

$$
\begin{aligned}
z_{n} & =w_{n}+\sum_{i=0}^{L_{0}} h_{i} x_{n-i}+h_{002} x_{n}^{2} x_{n-2}^{*}+h_{330} x_{n-3}^{2} x_{n}^{*} \\
& +h_{001} x_{n}^{2} x_{n-1}^{*}+h_{003} x_{n}^{2} x_{n-3}^{*}+h_{110} x_{n-1}^{2} x_{n}^{*}
\end{aligned}
$$

We investigate the performance of the two implementations of the Volterra MMSE turbo equalizer. Information bits are encoded by a convolutional code with generating polynomials in octal $g_{1}=5$ and $g_{2}=7$ with trellis termination. The coded bits are then passed through a random interleaver and mapped into 8PSK symbols. At the receiver, the MMSE filters are set to have 9 taps $N_{1}=5$ and $N_{2}=3$. The soft demapper output is deinterleaved and forwarded to a MAP decoder. The output of the decoder, is interleaved and passed to the equalizer.

Fig. 3 plots the BER performance for simulations up to 4 turboiterations for three different MMSE equalizers: the exact MMSE linear equalizer, the No-Apriori MMSE equalizer with the exact error variance (implementation-a) and the No-Apriori MMSE equalizer with a simplified error variance (implementations-b) as proposed in [12]. The BER performances of the MMSE equalizers improve with the number of iterations. An SNR gain up to $2 \mathrm{~dB}$ is achieved at $\mathrm{BER}=10^{-4}$ between the 1 st and 4th iteration of the exact MMSE. Besides, since all versions of the MMSE equalizer are fed with zero aprioris in the first iteration, the BER curves match as one can expect. More importantly, at high SNR values, the NA-MMSE implementation-a outperforms the NA-MMSE implementation-b since the $3 \mathrm{rd}$ order interference terms can no longer be neglected to compute the variance error in the soft demapper.

\section{CONCLUSION}

This paper provides a proper derivation of the linear MMSE turbo equalizer for the nonlinear Volterra channel including some approximate implementations. Unlike what was commonly thought in literature, the proposed linear turbo equalizer is shown to be able to cancel both linear and nonlinear interference. Theoritical study and BER results confirm that the linear MMSE Volterra outperforms some of the previously proposed "nonlinear" interference cancellers. 


\section{REFERENCES}

[1] ETSI EN 302307 V1.2.1 (2009-08), "Digital video broadcasting (DVB); second generation framing structure, channel coding and modulation systems for broadcasting, interactive services, news gathering and other broadband satellite applications (DVB-S2)," European Standard (Telecommunications series), 082009.

[2] C.E. Burnet and W.G. Cowley, "Performance analysis of turbo equalization for nonlinear channels," in International Symposium on Information Theory, 2005. ISIT 2005. Proceedings., sept. 2005, pp. $2026-2030$.

[3] N.H. Ngo, S.S. Pietrobon, S.A. Barbulescu, and C. Burnet, "Optimisation of 16APSK constellation with a new iterative decoder on the nonlinear channel," in Communications Theory Workshop, 2006. Proceedings. 7th Australian, feb. 2006, pp. $71-75$.

[4] S. Benedetto, E. Biglieri, and R. Daffara, "Modeling and performance evaluation of nonlinear satellite links-a volterra series approach," IEEE Transactions on Aerospace and Electronic Systems, vol. AES-15, no. 4, pp. 494 -507, july 1979.

[5] Y.T. Su, Mu-Chung Chiu, and Yen-Chih Chen, "Turbo equalization of nonlinear TDMA satellite signals," in Global Telecommunications Conference, 2002. GLOBECOM '02. IEEE, nov. 2002, vol. 3, pp. 2860 - 2864 vol.3.

[6] A. Gutierrez and W.E. Ryan, "Performance of volterra and MLSD receivers for nonlinear band-limited satellite systems," Communications, IEEE Transactions on, vol. 48, no. 7, pp. $1171-1177$, jul 2000.

[7] M. Tuchler and A.C. Singer, "Turbo equalization: An overview," IEEE Transactions on Information Theory, vol. 57, no. 2, pp. 920 -952, feb. 2011.

[8] C. Douillard, M. Jezequel, and C. Berrou, "Iterative correction of intersymbol interference: Turbo equalization," European Trans. Telecommun, vol. 6, no. 5, pp. 507511, Sept.Oct 1995.

[9] C. Laot, A. Glavieux, and J. Labat, "Turbo equalization: adaptive equalization and channel decoding jointly optimized," IEEE Journal on Selected Areas in Communications, vol. 19, no. 9, pp. $1744-1752$, sep 2001.

[10] G. Colavolpe and A. Piemontese, "Novel SISO detection algorithms for nonlinear satellite channels," Wireless Communications Letters, IEEE, vol. 1, no. 1, pp. 22 -25, february 2012.

[11] M. Tuchler, A.C. Singer, and R. Koetter, "Minimum mean squared error equalization using a priori information," IEEE Transactions on Signal Processing, vol. 50, no. 3, pp. 673 683, mar 2002.

[12] D. Ampeliotis, A.A. Rontogiannis, K. Berberidis, M. Papaleo, and G.E. Corazza, "Turbo equalization of non-linear satellite channels using soft interference cancellation," in Advanced Satellite Mobile Systems, 2008. ASMS 2008. 4th, aug. 2008, pp. $289-292$.

[13] D.N. Liu and M.P. Fitz, "Iterative equalization in non-linear satellite channels," in 7th International Symposium on Turbo Codes and Iterative Information Processing (ISTC), 2012, aug. 2012, pp. $220-224$.

[14] A.A.M. Saleh, "Frequency-independent and frequencydependent nonlinear models of TWT amplifiers," IEEE Transactions on Communications, vol. 29, no. 11, pp. 1715 -1720, november 1981.
[15] H. Vincent Poor, An introduction to signal detection and estimation (2nd ed.), Springer-Verlag New York, Inc., New York, NY, USA, 1994. 